\title{
High genetic diversity among extraintestinal Escherichia coli isolates in pullets and layers revealed by a longitudinal study
}

\author{
Surya Paudel ${ }^{1 *}$, Beatrix Stessl ${ }^{2}$, Claudia Hess ${ }^{1}$, Angelika Zloch ${ }^{1}$ and Michael Hess ${ }^{1}$
}

\begin{abstract}
Background: Various information about the genetic diversity of Escherichia coli isolates from chickens are available but a detailed epidemiological investigation based upon isolates obtained from interrelated pullet and layer flocks is still missing. Therefore, in the course of a longitudinal epidemiological study on pullets and layers, $144 \mathrm{E}$. coli isolates from chickens with or without pathological lesions of the reproductive tract were serotyped and genotyped with pulsed-field gel electrophoresis (PFGE). These isolates were collected during rearing, peak and at the end of production. The actual study is the first of its kind so as to elucidate genetic relatedness among extraintestinal $E$. coli isolated from chickens with varying pathological conditions in interrelated layer farms/flocks at different stages of rearing.
\end{abstract}

Results: Serotyping revealed that $63.19 \%$ of the isolates could not be assigned to any of the three serotypes tested whereas $30.55 \%$ of the isolates belonged to serotype O1:K1, 4.86 \% to O2:K1 and $1.38 \%$ to O78:K80. After macrorestriction digest with Xbal, $91.66 \%$ of the isolates were typeable resulting in 96 distinct PFGE profiles. Among them, five PFGE types included isolates collected from diseased chickens as well as from birds without pathological lesions. This finding shows that pathogenicity of $E$. coli in layers seems to be largely influenced by concurrent susceptibility factors. Furthermore, in six out of eight cases where two isolates were collected from each of eight birds, different PFGE types were found in the same or different organs of the same bird. The existence of predominant or persistent $E$. coli genotypes was only observed in two cases.

Conclusions: It is concluded that extraintestinal E. coli genotypes and serotypes in pullets and layers are heterogenous and also do not maintain a single clonality within the same bird. The facts that E. coli strains did not show any definite clonal population structure based on geographical region, age of the host and pathological lesions should have relevance in further epidemiological studies and control strategies.

Keywords: Escherichia coli, Longitudinal epidemiology, Pullets, Layers, Pulsed-field gel electrophoresis

\footnotetext{
* Correspondence: surya.paudel@vetmeduni.ac.at

'Department for Farm Animals and Veterinary Public Health, Clinic for Poultry

and Fish Medicine, University of Veterinary Medicine, Veterinärplatz 1, 1210

Vienna, Austria

Full list of author information is available at the end of the article
} 


\section{Background}

Escherichia coli isolates that are extraintestinal in nature are associated with the disease named colibacillosis that can infect all aged groups of chickens [1]. In layers, the pathogen is able to cause a systemic infection leading to fibrinous polyserositis, pericarditis, perihepatitis, salpingitis, peritonitis, salpingoperitonitis and a decrease in egg production ultimately leading to severe economic losses [2-8]. Despite serological diversities, serogroups such as $\mathrm{O} 1, \mathrm{O} 2$ and $\mathrm{O} 78$ are mostly implicated in disease conditions [9-11]. Until now, the pathogenicity of $E$. coli infection in chickens is not well understood. Several putative virulence and virulence-associated genes have been reported in avian pathogenic E. coli (APEC) $[1,11,12]$. However, the fact that a single genetic trait cannot separate disease-associated $E$. coli from commensal intestinal isolates raised certain concern on the definition of APEC as a single pathotype [13, 14].

From an epidemiological point of view, understanding the clonal population structure of extraintestinal $E$. coli involving a longitudinal sampling scheme in interrelated rearing and laying flocks has a high priority. Thus we performed a longitudinal study in order to characterize the relatedness among $E$. coli isolates from systemic organs of pullets and layers kept in alternative housing systems in Austria. Beside the determination of the serotype, pulsed-field gel electrophoresis (PFGE) was applied for genetic fingerprinting which has higher discriminating power compared to other methods such as multilocus sequence typing [15]. PFGE is more applicable to investigate large-scale genomic diversity within a distinct population and has also been previously applied to infer molecular relatedness among APEC isolates in other geographical locations [16-18].

\section{Methods}

\section{Flock history, sampling and $E$. coli isolation}

The present investigation was focused on extraintestinal E. coli isolates from pullets and laying hens kept in alternative husbandry system that were located in different provincial states of Austria. Six rearing and eight related layer farms comprising 15 layer flocks were included in the longitudinal study. Rearing farms are designated with letter "R" along with farm numbers as $R_{I}-R_{V I}$ (e. g. $R_{I}$ is rearing farm 1). The layer flocks are designated with letter " $\mathrm{L}$ " along with the flock number and the corresponding rearing farm (e. g. $\mathrm{L}_{1 / \mathrm{I}}$ indicate for layer flock 1 that comes from rearing farm 1). Detailed information on farms and flocks is provided in Table 1.

In total, 188 birds were sampled for extraintestinal $E$. coli based on the sampling scheme as shown in Fig. 1. Sampling was performed during rearing (age of birds: 16-19 weeks), at the peak of production (age of birds: 37-42 weeks) and at the end of production (age of birds: 64-80 weeks). In each of the sampling events, five birds per rearing farm/layer flock were necropsied and sampled for extraintestinal $E$. coli. In two flocks of one layer farm $\left(\mathrm{L}_{2 / \mathrm{IV}}\right.$ and $\left.\mathrm{L}_{3 / \mathrm{IV}}\right)$, additional samplings were included

Table 1 Farms and flocks included in the study

\begin{tabular}{|c|c|c|c|c|c|c|}
\hline \multicolumn{2}{|l|}{ Rearing farm } & \multicolumn{5}{|c|}{ Layer farm/flock } \\
\hline Farm identification $^{a}$ & Location & Farm & Flock identification $^{b}$ & Location & Housing system ${ }^{c}$ & Flock size $^{d}$ \\
\hline \multirow[t]{3}{*}{$\overline{R_{l}}$} & Lower Austria & 1 & $L_{1 / 1}$ & Styria & $\mathrm{FR}$ & 7500 \\
\hline & & & $L_{2 / 1}$ & Styria & $\mathrm{FR}$ & 3800 \\
\hline & & & $L_{3 / 1}$ & Styria & $\mathrm{DL}$ & 3440 \\
\hline \multirow[t]{2}{*}{$\mathrm{R}_{\|}$} & Salzburg & 2 & $\mathrm{~L}_{1 / \|}$ & Carinthia & ORG & 3000 \\
\hline & & 3 & $\mathrm{~L}_{2 / 11}$ & Lower Austria & ORG & 3000 \\
\hline \multirow[t]{3}{*}{$\mathrm{R}_{\text {III }}$} & Styria & 4 & $L_{1 / I I}$ & Burgenland & ORG & 6000 \\
\hline & & & $L_{2 / I I I}$ & Burgenland & ORG & 6000 \\
\hline & & & $L_{3 / I I I}$ & Burgenland & ORG & 6000 \\
\hline \multirow[t]{3}{*}{$\mathrm{R}_{\mathrm{IV}}$} & Upper Austria & 5 & $\mathrm{~L}_{1 / \mathrm{IV}}$ & Lower Austria & $\mathrm{DL}$ & 5980 \\
\hline & & & $\mathrm{L}_{2 / \mathrm{IV}}$ & Lower Austria & $\mathrm{DL}$ & 10890 \\
\hline & & & $L_{3 / I V}$ & Lower Austria & $\mathrm{DL}$ & 9030 \\
\hline \multirow[t]{2}{*}{$R_{V}$} & Styria & 6 & $\mathrm{~L}_{1 N}$ & Styria & $\mathrm{DL}$ & 17738 \\
\hline & & 7 & $L_{2 N}$ & Styria & $\mathrm{DL}$ & 14950 \\
\hline \multirow[t]{2}{*}{$\mathrm{R}_{\mathrm{VI}}$} & Burgenland & 8 & $\mathrm{~L}_{1 \mathrm{~N}}$ & Styria & $\mathrm{DL}$ & 2950 \\
\hline & & & $\mathrm{L}_{2 \mathrm{NI}}$ & Styria & $\mathrm{FR}$ & 7300 \\
\hline
\end{tabular}

${ }^{a}$ six rearing farms are indicated as $R_{I}-R_{V I}$ (all birds were kept in deep litter system)

blayer flocks are designated with letter " $L$ " along with flock number/corresponding rearing farm number

chousing system: FR - conventional free range, ORG - organic free range, DL - deep litter

${ }^{d}$ number of birds in each layer flock 


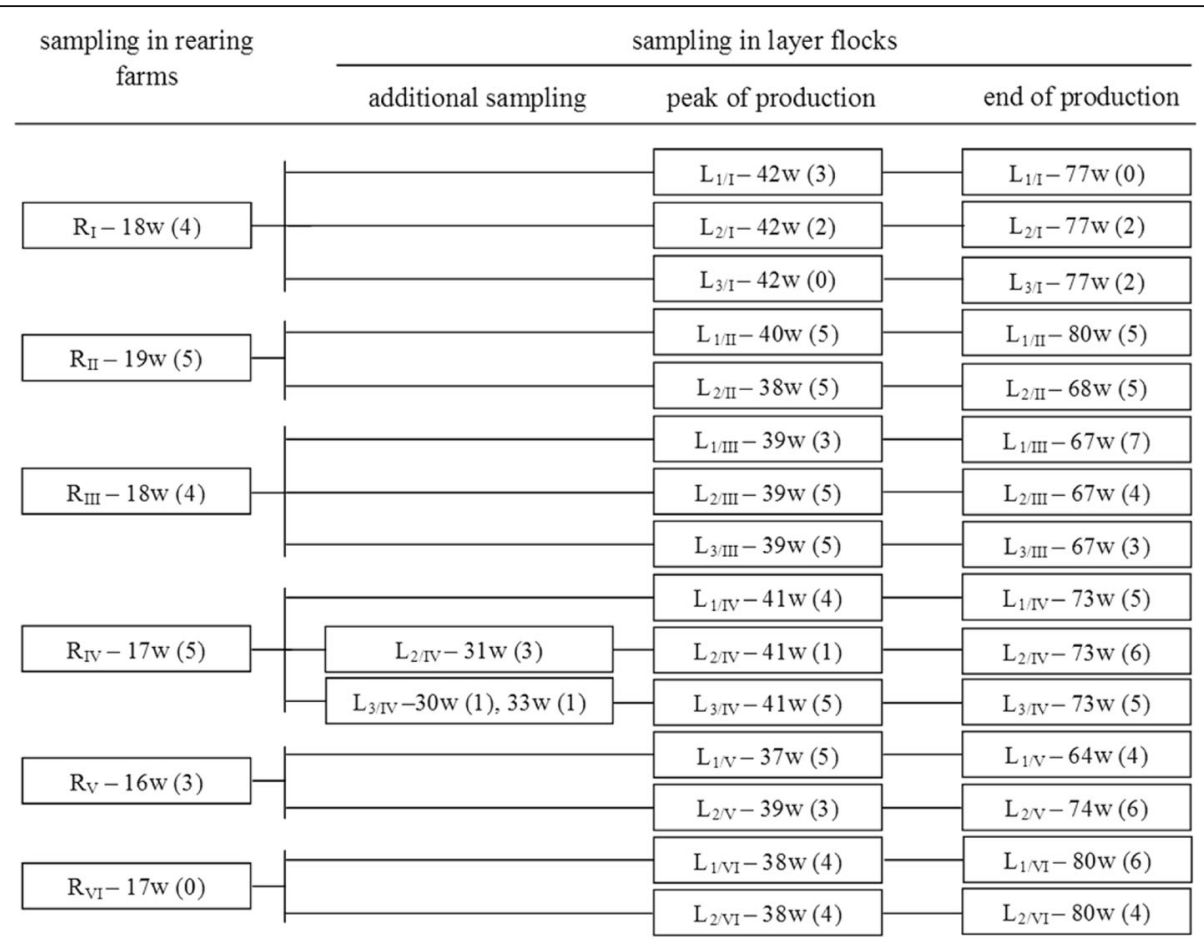

Fig. 1 Sampling scheme for the longitudinal study of extraintestinal Escherichia coli in pullets and layers. Each box represents an individual sampling event and includes the following information: farm/flock identification (rearing farms are indicated as $R_{1}-R_{V I}$ and layer flocks are designated with letter "L" along with flock number/corresponding rearing farm number) - age of birds during sampling (weeks) - number of E. coli isolates in parenthesis

at 30-33 weeks of age (eight birds in total) because of increased mortality and drop in egg production. The sampling scheme was focused on the isolation of $E$. coli from the reproductive organs (ovary and oviduct). Where E. coli could not be isolated from the reproductive tract, isolates from liver, heart or lung were chosen for further investigation. For isolation of E. coli, organ samples were aseptically streaked on McConkey agar (Scharlau, Vienna, Austria) and incubated at $37{ }^{\circ} \mathrm{C}$ for $24 \mathrm{~h}$ aerobically. On the following day, subcultures were made on Columbia agar supplemented with $5 \%$ sheep blood (COS agar, BioMérieux, Vienna, Austria) and incubated at $37{ }^{\circ} \mathrm{C}$ for $24 \mathrm{~h}$ aerobically. Most of the isolates were collected from ovary or oviduct (number of isolates $n=106)$ followed by liver $(n=25)$, lung $(n=10)$ and heart $(n=3)$. Details on $E$. coli isolates included in the present study are shown in Table 2. Isolates from rearing farms are marked with letter " $R$ " along with farm number and bird number (e. g. $\mathrm{R}_{\mathrm{I}}-1$ denotes for $E$. coli isolate collected from rearing farm 1 and bird number 1). Likewise, isolates from layer flocks are labelled with letter "L" along with flock number/corresponding rearing farm number - time of sampling (A: peak of production, B: end of production, Z1 or Z2: first or second additional samplings) - bird number - organs (only in those birds from where two samples were collected). For instance, $\mathrm{L}_{1 / \mathrm{I}}-\mathrm{A}-1$ denotes for the isolate collected from layer flock 1 that originated from rearing farm 1; at the peak of production; bird number 1. Generally, one $E$. coli isolate per bird was included for further characterization. However, in the case of eight birds, two isolates per bird from the same or different organs were collected at the same sampling event: $\mathrm{L}_{1 / \mathrm{III}}-\mathrm{B}-2$-ovary1, $\mathrm{L}_{1 / \mathrm{III}}$ - $\mathrm{B}$-2-ovary2; $\mathrm{L}_{1 / \mathrm{III}}-\mathrm{B}$-3-ovary, $\mathrm{L}_{1 / \mathrm{III}}-\mathrm{B}$-3-oviduct; $\mathrm{L}_{1 / \mathrm{III}}{ }^{-}$ B-4-oviduct1, $\mathrm{L}_{1 / \mathrm{III}}-\mathrm{B}-4$-oviduct2; $\mathrm{L}_{2 / \mathrm{IV}}$-B-1-ovary, $\mathrm{L}_{2 / \mathrm{IV}}$-B1-liver; $\mathrm{L}_{1 / \mathrm{V}^{-}} \mathrm{A}$-4-oviduct, $\mathrm{L}_{1 / \mathrm{V}^{-}} \mathrm{A}-4$-ovary; $\mathrm{L}_{2 / \mathrm{v}^{-}} \mathrm{B}-5$-ovary, $\mathrm{L}_{2 / \mathrm{v}^{-}} \mathrm{B}-5$-heart, $\mathrm{L}_{1 / \mathrm{VI}}-\mathrm{B}-1$-ovary, $\mathrm{L}_{1 / \mathrm{VI}}-\mathrm{B}-1$-oviduct; $\mathrm{L}_{2 / \mathrm{VI}^{-}}$ B-2-ovary, $\mathrm{L}_{2 / \mathrm{NI}}$-B-2-oviduct.

Additionally, gross pathological lesions of the reproductive tract were recorded. Pullets from all six rearing farms did not show any gross pathological lesions. At the peak of production, some E. coli isolates originated from birds showing lesions in the reproductive tract, including egg peritonitis, inflammation of ovary and/or oviduct and degeneration of ovary and/or oviduct in 5 , 17 and 7 birds respectively. Also, at the end of production, egg peritonitis, inflammation of ovary and/or oviduct and degeneration of ovary and/or oviduct were recorded in 15, 40 and 9 birds, respectively. In additional samplings, gross pathological lesions found in the 
Table 2 E. coli isolates and pathological findings in reproductive tract

\begin{tabular}{|c|c|c|c|c|c|}
\hline Farm/flock & Isolate identification $^{\text {a }}$ & Age (weeks) & Reproductive lesions & Serotype & PFGE type \\
\hline \multicolumn{6}{|c|}{ Rearing farm 1} \\
\hline \multirow[t]{4}{*}{$\mathrm{R}_{1}$} & $R_{1}-1$ & 18 & no & $n t^{b}$ & LA23 \\
\hline & $\mathrm{R}_{\mathrm{r}}-2$ & & no & $\mathrm{nt}$ & $\mathrm{nt}$ \\
\hline & $\mathrm{R}_{\mathrm{l}}-3$ & & no & $\mathrm{nt}$ & LA20 \\
\hline & $\mathrm{R}_{1}-4$ & & no & nt & LA19 \\
\hline \multirow[t]{3}{*}{$\mathrm{L}_{1 / 1}-\mathrm{A}$} & $\mathrm{L}_{1 / /}-\mathrm{A}-1$ & 42 & no & nt & S31 \\
\hline & $\mathrm{L}_{1 / /}-\mathrm{A}-2$ & & no & $\mathrm{nt}$ & S37 \\
\hline & $\mathrm{L}_{1 / 1}-\mathrm{A}-5$ & & no & O78:K80 & S6 \\
\hline \multirow[t]{2}{*}{$\mathrm{L}_{2 / 1}-\mathrm{A}$} & $\mathrm{L}_{2 / 1^{-}} \mathrm{A}-1$ & 42 & no & O1:K1 & S2 \\
\hline & $\mathrm{L}_{2 / 1}-\mathrm{A}-5$ & & no & $\mathrm{O} 1: \mathrm{K} 1$ & S15 \\
\hline \multirow[t]{2}{*}{$\mathrm{L}_{2 / 1}-\mathrm{B}$} & $\mathrm{L}_{2 / /}-\mathrm{B}-4$ & 77 & oophoritis & $\mathrm{nt}$ & S22 \\
\hline & $L_{2 / /}-B-5$ & & oophoritis & $\mathrm{nt}$ & S23 \\
\hline \multirow[t]{2}{*}{$\mathrm{L}_{3 / 1}-\mathrm{B}$} & $\mathrm{L}_{3 / 1}-\mathrm{B}-1$ & 77 & oophoritis and salpingitis & $\mathrm{O} 2: \mathrm{K} 1$ & S19 \\
\hline & $\mathrm{L}_{3 / /}-\mathrm{B}-2$ & & oophoritis & $\mathrm{O} 2: \mathrm{K} 1$ & S19 \\
\hline \multicolumn{6}{|c|}{ Rearing farm 2} \\
\hline \multirow[t]{5}{*}{$\mathrm{R}_{\|}$} & $\mathrm{R}_{\|^{-1}}-1$ & 19 & no & $\mathrm{O} 1: \mathrm{K} 1, \mathrm{O} 2: \mathrm{K} 1,078: \mathrm{K} 80$ & $\mathrm{Sa} 3$ \\
\hline & $\mathrm{R}_{\|}-2$ & & no & $\mathrm{O} 1: \mathrm{K} 1, \mathrm{O} 2 \mathrm{~K} 1$ & Sa1 \\
\hline & $\mathrm{R}_{\|}-3$ & & no & nt & $\mathrm{Sa} 4$ \\
\hline & $\mathrm{R}_{\|}-4$ & & no & O1:K1 & Sa5 \\
\hline & $R_{\|}-5$ & & no & nt & $\mathrm{Sa} 2$ \\
\hline \multirow[t]{5}{*}{$\mathrm{L}_{1 / 1 /} \mathrm{A}$} & $\mathrm{L}_{1 / 11}-\mathrm{A}-1$ & 40 & no & O1:K1 & $\mathrm{nt}$ \\
\hline & $\mathrm{L}_{1 / 11}-\mathrm{A}-2$ & & no & O1:K1 & $\mathrm{nt}$ \\
\hline & $\mathrm{L}_{1 / 1 / 1}-\mathrm{A}-3$ & & no & $\mathrm{O} 1: \mathrm{K} 1$ & $\mathrm{nt}$ \\
\hline & $\mathrm{L}_{1 / 1}-\mathrm{A}-4$ & & no & $\mathrm{O} 1: \mathrm{K} 1$ & $\mathrm{nt}$ \\
\hline & $\mathrm{L}_{1 / 11}-\mathrm{A}-5$ & & no & $\mathrm{O} 1: \mathrm{K} 1$ & $\mathrm{nt}$ \\
\hline \multirow[t]{5}{*}{$\mathrm{L}_{2 / I^{-}} \mathrm{A}$} & $\mathrm{L}_{2 / 1}-\mathrm{A}-1$ & 38 & degeneration of oviduct & $\mathrm{O} 1: \mathrm{K} 1, \mathrm{O} 2: \mathrm{K} 1$ & $\mathrm{nt}$ \\
\hline & $\mathrm{L}_{2 / 1}-\mathrm{A}-2$ & & no & $\mathrm{O} 1: \mathrm{K} 1, \mathrm{O} 2: \mathrm{K} 1$ & LA10 \\
\hline & $\mathrm{L}_{2 / 1}-\mathrm{A}-3$ & & oophoritis & $\mathrm{O} 2: \mathrm{K} 1$ & LA6 \\
\hline & $\mathrm{L}_{2 / 1}-\mathrm{A}-4$ & & oophoritis & $\mathrm{O} 1: \mathrm{K} 1, \mathrm{O} 2: \mathrm{K} 1, \mathrm{O} 78: \mathrm{K} 80$ & LA9 \\
\hline & $\mathrm{L}_{2 / 1} 1^{-A-5}$ & & oophoritis & O1:K1,O2:K1, & LA2 \\
\hline \multirow[t]{5}{*}{$L_{1 / I^{-}} B$} & $L_{1 / 11}-B-1$ & 80 & egg peritonitis & $\mathrm{nt}$ & $\mathrm{Ca} 4$ \\
\hline & $\mathrm{L}_{1 / 11} \mathrm{~B}-2$ & & no & $\mathrm{O} 2: \mathrm{K} 1$ & $\mathrm{Ca} 2$ \\
\hline & $L_{1 / 11}-B-3$ & & egg peritonitis & $\mathrm{nt}$ & Ca3 \\
\hline & $\mathrm{L}_{1 / 11}-\mathrm{B}-4$ & & egg peritonitis & nt & $\mathrm{Ca} 5$ \\
\hline & $L_{1 / 11}-B-5$ & & egg peritonitis & nt & Ca1 \\
\hline \multirow[t]{5}{*}{$\mathrm{L}_{2 / 1} 1^{-B}$} & $L_{2 / 11}-B-1$ & 68 & oophoritis & $\mathrm{nt}$ & LA17 \\
\hline & $\mathrm{L}_{2 / \|} \mathrm{B}-2$ & & oophoritis & nt & LA18 \\
\hline & $L_{2 / 11}-B-3$ & & oophoritis & $\mathrm{nt}$ & LA18 \\
\hline & $\mathrm{L}_{2 / \|} \mathrm{B}-4$ & & oophoritis & nt & LA7 \\
\hline & $L_{2 / 1}-B-5$ & & oophoritis & $\mathrm{nt}$ & LA18 \\
\hline \multicolumn{6}{|c|}{ Rearing farm 3} \\
\hline \multirow[t]{4}{*}{$\mathrm{R}_{\mathrm{III}}$} & $R_{\| I I}-1$ & 18 & no & $\mathrm{nt}$ & S16 \\
\hline & $R_{\| I I}-2$ & & no & $\mathrm{nt}$ & S5 \\
\hline & $\mathrm{R}_{\mathrm{III}}-3$ & & no & $\mathrm{nt}$ & S5 \\
\hline & $\mathrm{R}_{\| 11}-4$ & & no & $\mathrm{O} 1: \mathrm{K} 1, \mathrm{O} 2 \mathrm{~K} 1$ & S4 \\
\hline
\end{tabular}


Table 2 E. coli isolates and pathological findings in reproductive tract (Continued)

\begin{tabular}{|c|c|c|c|c|c|}
\hline \multirow{3}{*}{$\mathrm{L}_{1 / 111}-\mathrm{A}$} & $\mathrm{L}_{1 / 111}-\mathrm{A}-1$ & 39 & oophoritis & $\mathrm{nt}$ & $\mathrm{B} 15$ \\
\hline & $\mathrm{L}_{1 / 111}-\mathrm{A}-3$ & & no & $\mathrm{O} 1: \mathrm{K} 1$ & B14 \\
\hline & $L_{1 / \| 11}-A-5$ & & oophoritis & $\mathrm{nt}$ & B3 \\
\hline \multirow[t]{5}{*}{$\mathrm{L}_{2 / 11}-\mathrm{A}$} & $\mathrm{L}_{2 / \| 11}-\mathrm{A}-1$ & 39 & no & $\mathrm{nt}$ & B4 \\
\hline & $\mathrm{L}_{2 / \| 11}-\mathrm{A}-2$ & & no & $\mathrm{O} 1: \mathrm{K} 1$ & B6 \\
\hline & $L_{2 / 11}-A-3$ & & no & $\mathrm{nt}$ & B4 \\
\hline & $\mathrm{L}_{2 / 111}-\mathrm{A}-4$ & & no & $\mathrm{nt}$ & B8 \\
\hline & $L_{2 / \| 11}-A-5$ & & no & $\mathrm{nt}$ & B8 \\
\hline \multirow[t]{5}{*}{$\mathrm{L}_{3 / 111} \mathrm{~A}$} & $\mathrm{~L}_{3 / 11}-\mathrm{A}-1$ & 39 & oophoritis & $\mathrm{nt}$ & B1 \\
\hline & $\mathrm{L}_{3 / \| 1}-\mathrm{A}-2$ & & oophoritis & $\mathrm{nt}$ & B1 \\
\hline & $\mathrm{L}_{3 / 111}-\mathrm{A}-3$ & & oophoritis & $\mathrm{nt}$ & B1 \\
\hline & $\mathrm{L}_{3 / 111}-\mathrm{A}-4$ & & oophoritis & $\mathrm{nt}$ & B1 \\
\hline & $L_{3 / 111}-A-5$ & & oophoritis & $\mathrm{nt}$ & $\mathrm{B} 17$ \\
\hline \multirow[t]{7}{*}{$\mathrm{L}_{1 / \|} \|^{-B}$} & $L_{1 / 111}-B-2-o v a r y 1$ & 67 & egg peritonitis & $\mathrm{O} 1 \mathrm{~K} 1$ & $\mathrm{~B} 13$ \\
\hline & $\mathrm{L}_{1 / 111}-\mathrm{B}-2$-ovary2 & & egg peritonitis & $\mathrm{nt}$ & $\mathrm{B} 12$ \\
\hline & 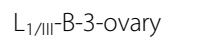 & & no & $\mathrm{O} 1 \mathrm{~K} 1$ & B12 \\
\hline & $\mathrm{L}_{1 / 111}-\mathrm{B}$-3-oviduct & & no & $\mathrm{nt}$ & $\mathrm{B} 12$ \\
\hline & $\mathrm{L}_{1 / 111} \mathrm{~B}-4$-oviduct1 & & no & $\mathrm{nt}$ & B5 \\
\hline & $\mathrm{L}_{1 / \| I I} \mathrm{~B}-4$-oviduct2 & & no & $\mathrm{nt}$ & B11 \\
\hline & $\mathrm{L}_{1 / \| 11}-\mathrm{B}-5$ & & degeneration of ovary and oviduct & nt & $\mathrm{B} 10$ \\
\hline \multirow[t]{4}{*}{$\mathrm{L}_{2 / \|} \|^{-B}$} & $L_{2 / \| I}-B-1$ & 67 & no & $\mathrm{O} 1 \mathrm{~K} 1$ & B7 \\
\hline & $L_{2 / 11}-B-2$ & & egg peritonitis & $\mathrm{O}: \mathrm{K} 1$ & B7 \\
\hline & $L_{2 / 111}-B-3$ & & degeneration of ovary and oviduct & $\mathrm{O}: \mathrm{K} 1$ & B9 \\
\hline & $L_{2 / 111}-B-4$ & & egg peritonitis & $\mathrm{O}: \mathrm{K} 1$ & B9 \\
\hline \multirow[t]{3}{*}{$\mathrm{L}_{3 / 111^{-}} \mathrm{B}$} & $L_{3 / 111}-B-3$ & 67 & no & $\mathrm{O}: \mathrm{K} 1$ & B16 \\
\hline & $\mathrm{L}_{3 / 111}-\mathrm{B}-4$ & & oophoritis & $\mathrm{O}: \mathrm{K} 1$ & B2 \\
\hline & $L_{3 / 111}-B-5$ & & no & $\mathrm{O} 1: \mathrm{K} 1$ & $\mathrm{nt}$ \\
\hline \multicolumn{6}{|c|}{ Rearing farm 4} \\
\hline \multirow[t]{5}{*}{$\mathrm{R}_{\mathrm{IV}}$} & $R_{\mathbb{N}^{-1}}$ & 17 & no & $\mathrm{nt}$ & Ua2 \\
\hline & $\mathrm{R}_{\mathrm{IV}}-2$ & & no & $\mathrm{nt}$ & Ua1 \\
\hline & $R_{I^{-}-3}$ & & no & $\mathrm{nt}$ & Ua1 \\
\hline & $\mathrm{R}_{\mathbb{I}}-4$ & & no & $\mathrm{nt}$ & Ua3 \\
\hline & $\mathrm{R}_{\mathbb{I} V^{-5}}$ & & no & $\mathrm{nt}$ & Ua4 \\
\hline \multirow[t]{4}{*}{$\mathrm{L}_{1 / \mathbb{V}-\mathrm{A}}$} & $\mathrm{L}_{1 / \mathrm{IV}}-\mathrm{A}-1$ & 41 & no & $\mathrm{nt}$ & LA16 \\
\hline & $\mathrm{L}_{1 / \mathrm{IV}}-\mathrm{A}-2$ & & no & $\mathrm{nt}$ & LA15 \\
\hline & $\mathrm{L}_{1 / \mathrm{IV}}-\mathrm{A}-3$ & & no & nt & LA15 \\
\hline & $\mathrm{L}_{1 / \mathbb{V} \mathrm{V}}-\mathrm{A}-4$ & & no & $\mathrm{nt}$ & LA27 \\
\hline $\mathrm{L}_{2 / \mathrm{V}^{-} \mathrm{A}}$ & $\mathrm{L}_{2 / \mathrm{IV}}-\mathrm{A}-2$ & 41 & no & $\mathrm{O} 1: \mathrm{K} 1$ & LA13 \\
\hline \multirow[t]{5}{*}{$L_{3 / \mathbb{V}}-A$} & $\mathrm{~L}_{3 / \mathrm{IV}}-\mathrm{A}-1$ & 41 & degeneration of ovary and oviduct & $\mathrm{nt}$ & LA21 \\
\hline & $\mathrm{L}_{3 / \mathbb{V}}-\mathrm{A}-2$ & & egg peritonitis & $\mathrm{nt}$ & LA21 \\
\hline & $\mathrm{L}_{3 / \mathrm{IV}}-\mathrm{A}-3$ & & degeneration of ovary and oviduct & $\mathrm{nt}$ & LA11 \\
\hline & $\mathrm{L}_{3 / \mathrm{IV}}-\mathrm{A}-4$ & & degeneration of ovary and oviduct & $\mathrm{nt}$ & LA11 \\
\hline & $\mathrm{L}_{3 / \mathrm{IV}}-\mathrm{A}-5$ & & no & nt & LA4 \\
\hline \multirow[t]{2}{*}{$\mathrm{L}_{1 / \mathbb{N}-\mathrm{B}}$} & $L_{1 / I V}-B-1$ & 73 & degeneration of ovary and oviduct & $\mathrm{nt}$ & LA25 \\
\hline & $\mathrm{L}_{1 / \mathrm{IV}}-\mathrm{B}-2$ & & oophoritis & $\mathrm{nt}$ & $\mathrm{LA} 1$ \\
\hline
\end{tabular}


Table 2 E. coli isolates and pathological findings in reproductive tract (Continued)

\begin{tabular}{|c|c|c|c|c|c|}
\hline & $L_{1 / I V}-B-3$ & & oophoritis & $\mathrm{nt}$ & $\mathrm{nt}$ \\
\hline & $\mathrm{L}_{1 / \mathbb{V} \mathrm{V}}-\mathrm{B}-4$ & & oophoritis & $\mathrm{nt}$ & LA5 \\
\hline & $L_{1 / \mathbb{I}}-B-5$ & & oophoritis & $\mathrm{nt}$ & LA25 \\
\hline \multirow[t]{6}{*}{$\mathrm{L}_{2 / 1 \mathrm{~V}^{-} \mathrm{B}}$} & $\mathrm{L}_{2 / \mathrm{V}}-\mathrm{B}-1$-ovary & 73 & oophoritis & nt & LA25 \\
\hline & $L_{2 / \mathbb{V}-B-1-l i v e r}$ & & oophoritis & O1:K1 & LA25 \\
\hline & $\mathrm{L}_{2 / \mathrm{I}}-\mathrm{B}-2$ & & oophoritis & O1:K1 & LA25 \\
\hline & $L_{2 / I V}-B-3$ & & oophoritis & $\mathrm{O} 1: \mathrm{K} 1$ & LA26 \\
\hline & $L_{2 / I V}-B-4$ & & oophoritis & O1:K1 & LA25 \\
\hline & $L_{2 / \mathbb{N}}-B-5$ & & oophoritis & $\mathrm{nt}$ & LA28 \\
\hline \multirow[t]{5}{*}{$\mathrm{L}_{3 / \mathbb{V}}-\mathrm{B}$} & $L_{3 / \mathbb{V}}-B-1$ & 73 & oophoritis & $\mathrm{O} 1: \mathrm{K} 1$ & LA3 \\
\hline & $L_{3 / \mathbb{V}}-B-2$ & & oophoritis & O1:K1 & LA14 \\
\hline & $L_{3 / \mathbb{V}}-B-3$ & & oophoritis & $\mathrm{O} 1: \mathrm{K} 1$ & LA25 \\
\hline & $\mathrm{L}_{3 / \mathrm{IV}^{-}-\mathrm{B}-4}$ & & oophoritis & nt & LA8 \\
\hline & $\mathrm{L}_{3 / \mathbb{V}}-\mathrm{B}-5$ & & oophoritis & O1:K1 & LA25 \\
\hline \multirow[t]{3}{*}{$\mathrm{L}_{2 / \mathbb{V}}-\mathrm{Z1}$} & $L_{2 / \mathbb{V}}-Z 1-1$ & 31 & oophoritis & $\mathrm{O} 1: \mathrm{K} 1, \mathrm{O} 2: \mathrm{K} 1,078: \mathrm{K} 80$ & LA22 \\
\hline & $L_{2 / \mathbb{V}}-Z 1-2$ & & no & O1:K1,O2:K1,O78:K80 & LA22 \\
\hline & $L_{2 / \mathbb{V}}-Z 1-3$ & & egg peritonitis & $\mathrm{O} 1: \mathrm{K} 1, \mathrm{O} 2: \mathrm{K} 1,078: \mathrm{K} 80$ & LA22 \\
\hline $\mathrm{L}_{3 / \mathrm{IV}-\mathrm{Z} 1}$ & $L_{3 / \mathbb{V}}-Z 1-2$ & 30 & degeneration of ovary and oviduct & $\mathrm{O} 1: \mathrm{K} 1, \mathrm{O}: \mathrm{K} 1$ & LA24 \\
\hline $\mathrm{L}_{3 / \mathrm{V}}-\mathrm{Z} 2$ & $\mathrm{~L}_{3 / \mathrm{V}}-\mathrm{Z2}-1$ & 33 & no & nt & LA12 \\
\hline \multicolumn{6}{|c|}{ Rearing farm 5} \\
\hline \multirow[t]{3}{*}{$\mathrm{R}_{\mathrm{v}}$} & $\mathrm{R}_{v}-3$ & 16 & no & $\mathrm{O} 1: \mathrm{K} 1, \mathrm{O} 2: \mathrm{K} 1$ & nt \\
\hline & $\mathrm{R}_{\mathrm{V}}-4$ & & no & O1:K1 & S7 \\
\hline & $\mathrm{R}_{v}-5$ & & no & $\mathrm{O} 1: \mathrm{K} 1$ & S7 \\
\hline \multirow[t]{5}{*}{$\mathrm{L}_{1 N}-\mathrm{A}$} & $\mathrm{L}_{1 / N}-\mathrm{A}-2$ & 37 & no & O1:K1 & $\mathrm{nt}$ \\
\hline & $\mathrm{L}_{1 / N}-\mathrm{A}-3$-oviduct & & no & nt & S1 \\
\hline & $\mathrm{L}_{1 / N}-\mathrm{A}-4$-oviduct & & egg peritonitis & nt & S18 \\
\hline & $\mathrm{L}_{1 / N}-\mathrm{A}-4$-ovary & & egg peritonitis & $\mathrm{O} 1: \mathrm{K} 1$ & S7 \\
\hline & $\mathrm{L}_{1 / N}-\mathrm{A}-6$ & & egg peritonitis & O1:K1 & $\mathrm{nt}$ \\
\hline \multirow[t]{4}{*}{$\mathrm{L}_{1 N}-\mathrm{B}$} & $L_{1 N}-B-1$ & 64 & egg peritonitis & $\mathrm{O} 2: \mathrm{K} 1$ & S8 \\
\hline & $\mathrm{L}_{1 / N}-\mathrm{B}-2$ & & egg peritonitis & $\mathrm{O} 2: \mathrm{K} 1$ & s9 \\
\hline & $L_{1 N}-B-3$ & & egg peritonitis & $\mathrm{nt}$ & S25 \\
\hline & $\mathrm{L}_{1 N} \sim \mathrm{B}-5$ & & egg peritonitis & $\mathrm{O} 2: \mathrm{K} 1$ & s9 \\
\hline \multirow[t]{3}{*}{$\mathrm{L}_{2 N} \mathrm{~A}$} & $\mathrm{~L}_{2 N}-\mathrm{A}-3$ & 39 & degeneration of ovary & nt & S27 \\
\hline & $\mathrm{L}_{2 N}-\mathrm{A}-4$ & & degeneration of ovary & O78:K80 & S29 \\
\hline & $L_{2 N}-A-5$ & & no & $\mathrm{nt}$ & S3 \\
\hline \multirow[t]{6}{*}{$\mathrm{L}_{2 N}-\mathrm{B}$} & $L_{2 N}-B-1$ & 74 & no & $\mathrm{O} 1: \mathrm{K} 1$ & $\mathrm{~S} 10$ \\
\hline & $\mathrm{L}_{2 N}-\mathrm{B}-2$ & & no & O1:K1 & $\mathrm{S} 10$ \\
\hline & $L_{2 N}-B-3$ & & oophoritis and salpingitis & $\mathrm{O} 1: \mathrm{K} 1$ & S10 \\
\hline & $\mathrm{L}_{2 N}-\mathrm{B}-4$ & & oophoritis & nt & S20 \\
\hline & $L_{2 N}-B-5$-ovary & & egg peritonitis & $\mathrm{O} 1: \mathrm{K} 1$ & S34 \\
\hline & $L_{2 N}-B-5$-heart & & egg peritonitis & O1:K1 & S34 \\
\hline \multicolumn{6}{|c|}{ Rearing farm 6} \\
\hline \multirow[t]{4}{*}{$\mathrm{L}_{1 N 1^{-}} \mathrm{A}$} & $\mathrm{L}_{1 / \mathrm{N} 1}-\mathrm{A}-1$ & 38 & no & nt & S36 \\
\hline & $\mathrm{L}_{1 / M^{-}}-\mathrm{A}-2$ & & no & $\mathrm{nt}$ & S21 \\
\hline & $\mathrm{L}_{1 / N^{1}}-\mathrm{A}-4$ & & oophoritis & $\mathrm{nt}$ & S11 \\
\hline & $\mathrm{L}_{1 / \mathrm{Vl}}-\mathrm{A}-5$ & & no & $\mathrm{nt}$ & S21 \\
\hline
\end{tabular}


Table 2 E. coli isolates and pathological findings in reproductive tract (Continued)

\begin{tabular}{|c|c|c|c|c|c|}
\hline \multirow[t]{6}{*}{$L_{1 / N 1^{-}} B$} & $\mathrm{~L}_{1 / N 1}-\mathrm{B}-1$-ovary & 80 & degeneration of ovary and oviduct & $\mathrm{O} 1: \mathrm{K} 1$ & S33 \\
\hline & $L_{1 / N 1}-B-1$-oviduct & & degeneration of ovary and oviduct & nt & $\mathrm{S} 12$ \\
\hline & $\mathrm{L}_{1 / N^{1}}-\mathrm{B}-2$ & & degeneration of ovary and oviduct & 01:K1 & S33 \\
\hline & $\mathrm{L}_{1 / N^{1}}-\mathrm{B}-3$ & & oophoritis & O1:K1 & S26 \\
\hline & $L_{1 / N 1}-B-4$ & & degeneration of ovary and oviduct & $\mathrm{nt}$ & S24 \\
\hline & $\mathrm{L}_{1 / \mathrm{N}^{\prime}} \mathrm{B}-\mathrm{-}-\mathrm{L}$ & & egg peritonitis & O1:K1 & S35 \\
\hline \multirow[t]{4}{*}{$\mathrm{L}_{2 N 1}-\mathrm{A}$} & $\mathrm{L}_{2 / \mathrm{N}^{-}}-\mathrm{A}-1$ & 38 & no & nt & S17 \\
\hline & $\mathrm{L}_{2 / N^{1}} \mathrm{~A}-2$ & & no & $\mathrm{nt}$ & S13 \\
\hline & $\mathrm{L}_{2 / \mathrm{N}^{1}} \mathrm{~A}-4$ & & oophoritis & $\mathrm{nt}$ & S32 \\
\hline & $\mathrm{L}_{2 N{ }^{1}}-\mathrm{A}-5$ & & oophoritis, degeneration of oviduct & nt & S17 \\
\hline \multirow[t]{4}{*}{$L_{2 N 1}-B$} & $\mathrm{~L}_{2 / \mathrm{M}}-\mathrm{B}-2$-ovary & 80 & oophoritis & nt & $\mathrm{S} 28$ \\
\hline & $\mathrm{L}_{2 \mathrm{~N}^{1}-\mathrm{B} \text {-2-oviduct }}$ & & oophoritis & O1:K1 & S30 \\
\hline & $L_{2 / M^{\prime}}-\mathrm{B}-4$ & & degeneration of ovary & $\mathrm{nt}$ & S14 \\
\hline & $\mathrm{L}_{2 / M^{1}} \mathrm{~B}-5$ & & egg peritonitis & O1:K1 & S32 \\
\hline
\end{tabular}

Age of birds, lesions in the reproductive tract, serotypes and PFGE types of each $E$. coli isolates are provided in the corresponding vertical line "isolates identification: isolates from rearing farms are marked with letter " $R$ " along with farm number and bird number. Likewise, isolates from layer flocks are labelled with letter "L" along with flock number/corresponding rearing farm number - time of sampling (A: peak of production, B: end of production, Z1 or Z2: first or second additional samplings) - bird number - organs (only in those birds from where two samples were collected)

${ }^{\mathrm{b}}$ non-typeable

reproductive tract comprised egg peritonitis in one bird, inflammation of ovary and/or oviduct in two birds and degeneration of ovary and oviduct in one bird.

\section{Subtyping of $E$. coli isolates}

Serotyping was performed on 144 E. coli isolates applying a slide agglutination test to Escherichia coli O1:K1, O2:K1 and O78:K80 antisera following supplier's guidelines (Animal Health and Veterinary Laboratory Agency, Weybridge, Surrey, UK).

For PFGE, E. coli isolates were grown on COS agar at $37{ }^{\circ} \mathrm{C}$ for $24 \mathrm{~h}$. The plug preparation and PFGE was performed according to the standardized Pulsenet International protocol for E. coli O157:H7, E. coli non-O157, Salmonella serotypes, Shigella sonnei and Shigella flexneri (http://www.pulsenetinternational.org/assets/Pulse Net/uploads/pfge/PNL05_Ec-Sal-ShigPFGEprotocol.pdf; accessed on 18.12.2015). The macrorestriction digest was performed applying XbaI (50 U/sample; Thermo Fisher Scientific, Fermentas; Waltham, Massachusetts, USA) at $37{ }^{\circ} \mathrm{C}$ for $2-3 \mathrm{~h}$. Restricted samples were separated in a $1 \%$ (w/v) SeaKem Gold agarose gel (Lonza Group AG, Basel, Switzerland) in $0.5 \times \mathrm{TBE}$ buffer at $6 \mathrm{~V} / \mathrm{cm}$ on a Chef DR ${ }_{\mathrm{II}} \mathrm{I}$ system (Bio-Rad Laboratories, Inc.). A linear ramping factor with pulse times from 2.2 to $54.2 \mathrm{~s}$ at $14{ }^{\circ} \mathrm{C}$ and an inclined angle of $120^{\circ}$ was applied for $22.5 \mathrm{~h}$. The gels were stained with ethidium bromide (Sigma Aldrich, Vienna, Austria), digitally photographed with Gel Doc 2000 (Bio-Rad Laboratories, Inc.) and normalized as TIFF images (BioNumerics 6.6 software Applied Math NV, SintMartens-Latem, Belgium) applying the PFGE global standard Salmonella ser. Braenderup H9812. In order to identify indistinguishable PFGE types, a Dice coefficient similarity of $100 \%$ was used.

\section{E. coli confirmation of non-typeable genotypes}

Partial sequencing of $16 \mathrm{~S}$ rRNA gene was done in PFGE non-typeable isolates $(n=12)$ as described previously [19]. For this purpose, strains were grown on COS agar plates at $37{ }^{\circ} \mathrm{C}$ for $24 \mathrm{~h}$. DNA extraction was done from two to three colonies using DNeasy Blood and Tissue Kit (QIAGEN, Hilden, Germany) following manufacturer's recommendation. PCR was performed with a set of primers: $16 \mathrm{~S}$ F 5'-GGCGGCRKGCCTAAYACATGC AAGT-3' and 16S R 5'-GACGACARCCATGCASC ACCTGT-3'. Amplification was carried out in $25 \mu \mathrm{l}$ reaction volume consisting of $12.5 \mu \mathrm{l}$ of HotStarTaq Master Mix (Qiagen, Hilden, Germany), $8 \mu$ l of nuclease free distilled water, $1 \mu \mathrm{l}$ of each forward and reverse primers $(10 \mathrm{pmol} / \mu \mathrm{l})$ and $2.5 \mu \mathrm{l}$ of DNA template. The PCR thermocycler was programmed as: initial denaturation at $95{ }^{\circ} \mathrm{C}$ for 15 min followed by 40 cycles of heat denaturation at $94{ }^{\circ} \mathrm{C}$, annealing at $60{ }^{\circ} \mathrm{C}$ for $1 \mathrm{~min}$ and extension at $72{ }^{\circ} \mathrm{C}$ for $1.5 \mathrm{~min}$. Final elongation was performed at $72{ }^{\circ} \mathrm{C}$ for $10 \mathrm{~min}$. The PCR products were visualized by agarose gel electrophoresis. The gel slices were cut and purified using QIAquick ${ }^{\circledR}$ gel extraction kit (QIAGEN, Germany). Samples were then dispatched to LGC genomics $\mathrm{GmbH}$ (Berlin, Germany) for sequencing. The data obtained were processed with software Accelrys Gene v2.5 (Accelrys Inc) and analyzed with BLAST search in NCBI database. 


\section{Antimicrobial resistance (AMR)}

Sixteen E. coli isolates originating from eight birds (two isolates per bird from the same or different organs) were investigated for the potential difference in AMR among strains isolated from the same organ (2 birds) or from different organs of the same bird ( 6 birds). The antimicrobial susceptibility test was performed using the disk diffusion method on Mueller-Hinton Agar (BioMeriéux, Vienna, Austria) according to Bauer et al. [20]). The following antimicrobials were tested: aminopenicilline [amoxicillin and ampicillin (each $10 \mu \mathrm{g}$ )], aminoglycoside [gentamicin $(10 \mu \mathrm{g})$, neomycin $(30 \mu \mathrm{g})]$, tetracyclines [tetracycline and doxycycline (each $30 \mu \mathrm{g})$ ], co-trimoxazole [sulphamethoxazole and trimethoprim $(25 \mu \mathrm{g})]$, macrolide (tylosin $30 \mu \mathrm{g})$, quinolone [oxolinic acid $2 \mu \mathrm{g}$, enrofloxacin $(5 \mu \mathrm{g})$ ], cephalosporine [ceftiofur $(30 \mu \mathrm{g})$ ], polymyxin [colistin $(10 \mu \mathrm{g})$ ] and aminocyclitol [spectinomycin $(100 \mu \mathrm{g})$ ]. Multidrug resistance (MDR) among avian E. coli was defined as resistance to three or more classes of antimicrobial agents.

\section{Results}

\section{Subtyping of $E$. coli isolates}

Serotyping revealed that 44 isolates (30.55\%) were grouped as O1:K1 while $7(4.86 \%)$ and $2(1.38 \%)$ strains belonged to O2:K1 and O78:K80, respectively. Furthermore, 91 isolates $(63.19 \%)$ could not be assigned to a definite serotype using these three antisera as they did not show agglutination $(n=79)$ or reacted positive with more than one anti-serum used $(n=12)$. Isolates that did not show agglutination with any or reacted positive with more than one anti-serum were assigned as nontypeable (Table 2). The PFGE analysis of $132 \mathrm{E}$. coli isolates resulted in a heterogenous PFGE cluster: $96 \mathrm{E}$. coli profiles were obtained after macrorestriction digest applying $X b a \mathrm{I}$ while 12 isolates were non-typeable. The dendrogram obtained from the cluster analysis is shown in Fig. 2.

The most abundant $E$. coli PFGE-profile was LA25 ( $n=$ 8) which included strains from three layer flocks $\left(\mathrm{L}_{1 / \mathrm{IV}}-\mathrm{B}\right.$, $\mathrm{L}_{2 / \mathrm{IV}}-\mathrm{B}$ and $\mathrm{L}_{3 / \mathrm{IV}}-\mathrm{B}$ ) that originated from a single rearing farm $\left(R_{I V}\right)$. All these isolates were associated with lesions in the reproductive tract. Likewise, B1 included four isolates from birds with inflammation of ovaries in the same flock $\left(\mathrm{L}_{3 / \mathrm{III}} \mathrm{A}\right)$. Furthermore, E. coli genotypes which caused reproductive tract lesions in more than one laying bird at one sampling occasion from the same flock were: B9 $(n=2)$, LA11 $(n=2)$, LA18 $(n=3)$, LA21 $(n=2)$, S19 $(n=2)$, S33 $(n=2)$, S34 $(n=2)$ and S9 $(n=2)$.

Interestingly, $E$. coli genotypes B7 $(n=2)$, B12 $(n=3)$, LA22 $(n=3), \mathrm{S} 10(n=3)$ and S17 $(n=2)$ included isolates from both normal and diseased chickens. S5 $(n=2)$, Ua1 $(n=2), \mathrm{B} 4(n=2), \mathrm{B} 8(n=2), \mathrm{LA} 15(n=2)$ and S21 $(n=2)$ were present in pullets or layers without clinical signs.
PFGE type S7 included three E. coli isolates that were collected from two pullets without pathological lesions and one laying hen with egg peritonitis originating from the same rearing farm.

\section{DNA sequencing}

The non-typable isolates were confirmed by partial sequencing of $16 \mathrm{~S}$ rRNA gene as E. coli (99-100 \% identity). Accession numbers of the isolates to the European $\mathrm{Nu}$ cleotide Archive are as follows: $\mathrm{R}_{\mathrm{I}-2}$ : LT548255, $\mathrm{L}_{1 / \mathrm{II}}-\mathrm{A}-1$ :

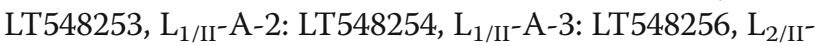
A-1: LT548257, $\mathrm{L}_{3 / \mathrm{III}}$-B-5: LT548258, $\mathrm{R}_{\mathrm{V}}$-3: LT548251, $\mathrm{L}_{1 / \mathrm{V}}$-A-2: LT548250, $\mathrm{L}_{1 / \mathrm{V}} \mathrm{A}-\mathrm{A}$ : LT548252. Following three isolates had $100 \%$ identity with the existing database: $\mathrm{L}_{1 / \mathrm{II}} \mathrm{A}-4$ : JQ975905.1, $\mathrm{L}_{1 / \mathrm{II}}$-A-5: JQ975905.1, $\mathrm{L}_{1 / \mathrm{IV}^{-}}$ B-3: KU560507.1.

\section{Antimicrobial resistance (AMR)}

The results of antibiotic resistance tests are shown in Table 3. These E. coli isolates were considered for the test in order to investigate similarities or differences in antibiotic sensitivity profiles between two strains collected from the same bird. All isolates were resistant to tylosin. Additionally, MDR was observed in three isolates originating from different birds. Two of these were resistant to five antibiotic substances \{aminopenicilline (amoxicillin and ampicillin), tetracycline, doxycycline, sulphamethoxazole and trimethoprim $\}$ and the other to three antibiotic substances (oxolinic acid, doxycycline and neomycin). The following pair of isolates had nonidentical pattern of resistance towards several antimicrobials used: 1) $\mathrm{L}_{1 / \mathrm{III}}-\mathrm{B}-2$-ovary 1 and $\mathrm{L}_{1 / \mathrm{III}}$-B-2-ovary2: amoxicillin; 2) $\mathrm{L}_{1 / \mathrm{III}}-\mathrm{B}-3$-ovary and $\mathrm{L}_{1 / \mathrm{III}}-\mathrm{B}-3$-oviduct: ampicillin, amoxycillin, doxycycline, tetracycline and sulphamethoxazole + trimethoprim; 3) $\mathrm{L}_{1 / \mathrm{III}}$-B-4-oviduct1 and $\mathrm{L}_{1 / \mathrm{III}}-\mathrm{B}-4$-oviduct 2 : ampicillin, amoxycillin, doxycycline, tetracycline and sulphamethoxazole + trimethoprim; 4) $\mathrm{L}_{2 / \mathrm{IV}}-\mathrm{B}-1$-ovary and $\mathrm{L}_{2 / \mathrm{IV}}-\mathrm{B}-1$-liver : amoxicillin; 5) $\mathrm{L}_{1 / \mathrm{V}} \mathrm{A}-4$-oviduct and $\mathrm{L}_{1 / \mathrm{V}} \mathrm{A}-4$-ovary : oxolinic acid; 6) $\mathrm{L}_{2 / \mathrm{V}}$-B-5-ovary and $\mathrm{L}_{2 / \mathrm{V}}$-B-5-heart : doxycycline, enrofloxacin, neomycin; 7) $\mathrm{L}_{1 / \mathrm{VI}}-\mathrm{B}-1$-ovary and $\mathrm{L}_{1 / \mathrm{VI}}-\mathrm{B}-1$-oviduct : oxolinic acid; 8) $\mathrm{L}_{2 / \mathrm{VI}}-\mathrm{B}$-2-ovary and $\mathrm{L}_{2 / \mathrm{VI}}-\mathrm{B}-2$-oviduct : amoxicillin and doxycycline.

\section{Discussion}

An infection with $E$. coli in layers is regarded as one of the major problems in global poultry industry that might cause reproductive disorders referred as salpingitis/ peritonitis/salpingoperitonitis and peritonitis syndrome ultimately leading to severe economic losses on commercial farms [6]. In this regards, an epidemiological knowledge of the disease and disease causing agent is fundamental in order to develop effective control and prophylactic strategies. Here, we studied molecular 


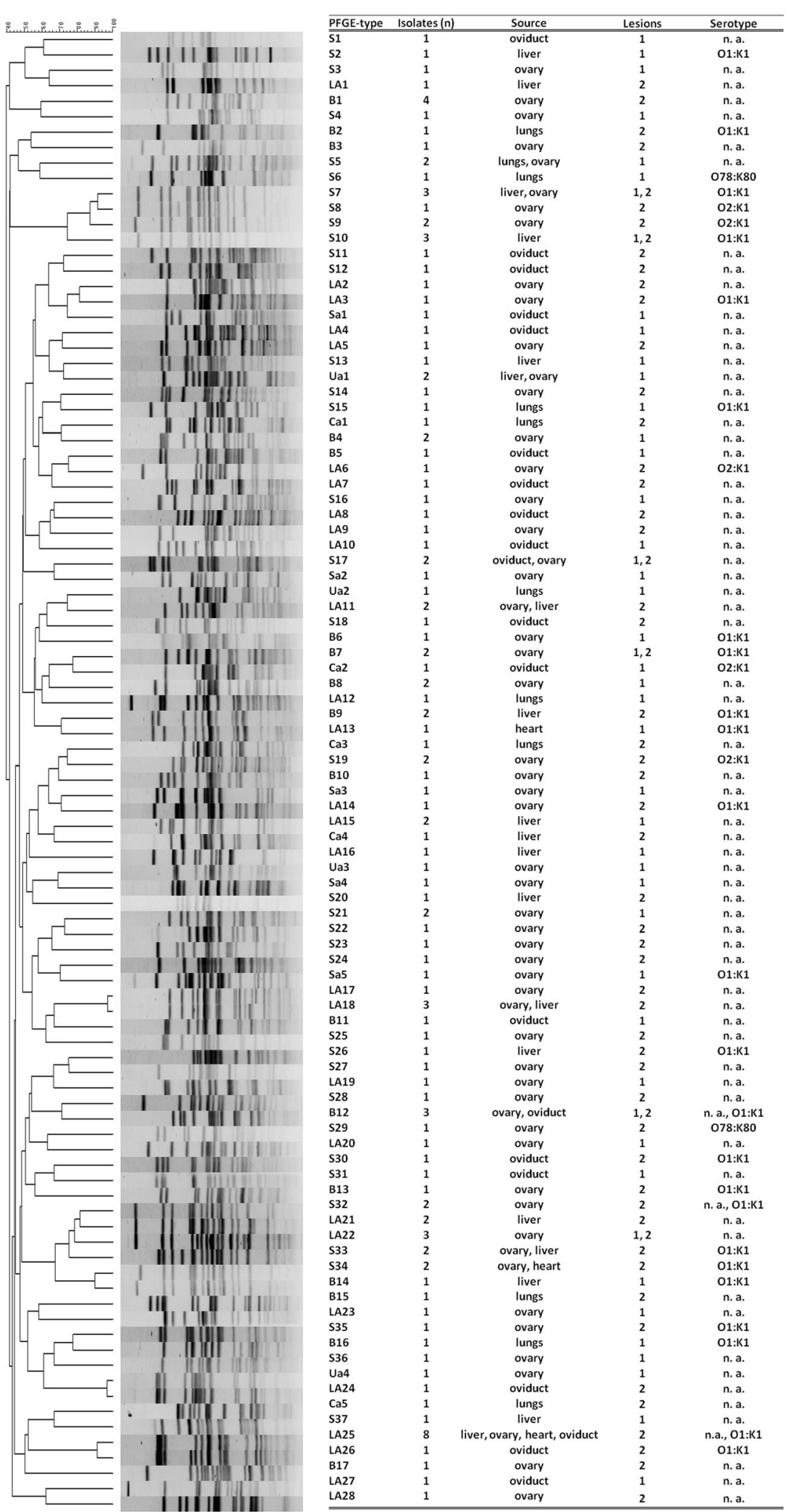

Fig. 2 (See legend on next page.) 
(See figure on previous page.)

Fig. 2 PFGE cluster analysis of Escherichia coli isolates from pullets and layers (restriction enzyme Xbal). The TIFF images were compared using BioNumerics 6.6 software (Applied Math NV, Sint-Martens-Latem, Belgium), and normalized using the PFGE global standard Salmonella ser. Braenderup H9812. Pattern clustering was performed using the unweighted pair group method using arithmetic averages (UPGMA) and the Dice correlation coefficient was applied with a position tolerance of $1.0 \%$. Information provided adjacent to the dendrogram include the PFGE-type in combination with areas of isolation (S: Styria, LA: Lower Austria, Ua: Upper Austria, Sa: Salzburg, B: Burgenland, Ca: Carinthia), number of isolates (n) in each PFGE type, organs of isolation (source) and serotype [not applicable (na) are untypeable isolates]. Furthermore, E. coli isolation was classified according to the absence (1) or presence (2) of lesions in the reproductive tract

epidemiology of $E$. coli isolates collected from pullets and layers in a longitudinal sampling study in Austria. Data obtained from genetic fingerprinting by PFGE were analyzed together with serotypes, geographical regions of isolation, and concurrent pathological lesions in each of the sampled birds.

In total, more than half of the $E$. coli isolates $(n=91 / 144)$ could not be assigned to a single serotype using antibodies against O1:K1, O2:K1 and O78:K80. Furthermore, for those isolates that could be assigned to one of the named serotypes, no correlation was found between a specific serotype and the occurrence of lesions in birds. In previous studies, it was also shown that E. coli isolates collected from diseased birds display a high serological diversity $[16,21,22]$, demonstrating as high as 62 different $\mathrm{O}$ serogroups [21]. Thus classifying $E$. coli strains into a definite serotype might sometimes be somewhat challenging. Hence, our finding is in agreement with a previous notion that serotyping alone might not be helpful as a tool for characterization of E. coli [16].

In this study, the PFGE subtyping of E. coli isolates $(n=132)$ resulted in $96 \mathrm{XbaI}$ profiles. Exclusively in two events, the same PFGE profile was seen in isolates from different sampling dates in mutually related farms/ flocks, indicating potential $E$. coli persistence. The PFGEtype S7 $(n=3)$ included isolates from pullets $(n=2$, rearing farm $R_{V}$ ) without pathological lesions and from one layer in the corresponding flock $\mathrm{L}_{1 / \mathrm{V}}$ suffering from egg peritonitis and fibrinous oophoritis at the peak of production. In the second case, PFGE type S32 contained two isolates from the same layer flock $\left(\mathrm{L}_{2 / \mathrm{VI}}\right)$ at the peak and end of production. One bird sampled at the peak of production showed inflammation of the ovary whereas egg peritonitis was diagnosed in the other birds necropsied at the end of production. These results indicate that some $E$. coli genotypes may retain in certain flocks at different stages of rearing but the associated pathological outcomes in birds can vary.

The genomic profile of extraintestinal $E$. coli with PFGE further revealed that strains collected from birds with pathological lesions can have $100 \%$ genetic identity with strains that were collected from healthy birds. For instance, in PFGE type $\mathrm{S} 10(n=3)$ in flock $\mathrm{L}_{2 / \mathrm{v}}$, two birds did not have any lesions while one had oophoritis and salpingitis. Likewise in PFGE type $\mathrm{B} 7(n=2)$ in $\mathrm{L}_{2 / \mathrm{III}}$, one bird showed no lesions while in contrast, the other had egg peritonitis. Also, remaining isolates could not be grouped into distinct clonal clusters based on presence or absence of pathological lesions in sampled birds. This finding is in agreement with a previous study in broilers where authors have reported a high heterogenecity of $E$. coli isolates in broilers $[13,23]$. It can be hypothesized that pathogenicity of extraintestinal $E$. coli in chickens is highly dependent on concurrent environmental and host susceptibility factors. Providing a suitable opportunity in certain circumstances, E. coli residing in clinically healthy chickens might turn up into pathogenic. The hypothesis is further supported by an earlier finding in broiler that many collibacillosis associated isolates might not be clearly distinguished solely on the basis of presence of virulence associated genes as compared to intestinal commensal E. coli [13].

In the present study, we found no evidence for clonality of $E$. coli with respect to geographical locations of farms. Previously, Ewers et al. (2004) found only a limited number of $E$. coli clones to be distributed in poultry production in Germany [16]. In another study, it was reported that chickens with peritonitis in a single flock were likely to be infected by the same E. coli strain [24]. Different to this, we did not find clonality of $E$. coli isolates in birds from the same flock showing gross pathological lesions in the reproductive tract thus maintaining a high heterogenicity of PFGE types. Interestingly, we further noticed that a single bird can harbour two different PFGE types of $E$. coli in the same or different organs. Thus, the study demonstrated that a layer can be infected simultaneously by different $E$. coli genotypes. A similar finding was previously reported in broilers [18]. However, in another study in layers, one PFGE type was found to be present in bone marrow of an individual bird [17]. It might be that in some organs $E$. coli isolates possess less or no genetic diversity due to an adaptation process, which should however be further elucidated. In the present study, we also tested antibiotic susceptibility of 16 isolates that were collected from eight birds. All the isolates were sensitive to ceftiofur, colistin, gentamicin and spectinomycin but the resistant rate to tylosin was found $100 \%$. Mixed results were obtained for other antibiotics tested. MDR was seen in 3/ 16 isolates showing resistance to as high as five different antibiotics used. Although the number of isolates included for antimicrobial susceptibility test in the actual study is 
Table 3 Antibiotic resistance test of 16 Escherichia coli isolates collected from 8 birds (two isolates per bird)

\begin{tabular}{|c|c|c|c|c|c|c|c|c|c|c|c|c|c|c|c|c|}
\hline \multirow[t]{2}{*}{ Antibiotics } & \multicolumn{16}{|l|}{ Isolates } \\
\hline & $\begin{array}{l}\text { L1/III-B-2- } \\
\text { ovary1 }\end{array}$ & $\begin{array}{l}\text { L1/III-B-2- } \\
\text { ovary2 }\end{array}$ & $\begin{array}{l}\text { L1/III-B- } \\
\text { 3-ovary }\end{array}$ & $\begin{array}{l}\text { L1/III-B-3- } \\
\text { oviduct }\end{array}$ & $\begin{array}{l}\text { L1/III-B-4- } \\
\text { oviduct1 }\end{array}$ & $\begin{array}{l}\text { L1/III-B-4- } \\
\text { oviduct2 }\end{array}$ & $\begin{array}{l}\text { L2/IV-B- } \\
1 \text {-ovary }\end{array}$ & $\begin{array}{l}\text { L2/IV-B- } \\
\text { 1-liver }\end{array}$ & $\begin{array}{l}\text { L1N-A-4- } \\
\text { oviduct }\end{array}$ & $\begin{array}{l}\text { L1N-A- } \\
\text { 4-ovary }\end{array}$ & $\begin{array}{l}\text { L2N-B- } \\
\text { 5-ovary }\end{array}$ & $\begin{array}{l}\text { L2N-B- } \\
\text { 5-heart }\end{array}$ & $\begin{array}{l}\text { L1/NI-B- } \\
\text { 1-ovary }\end{array}$ & $\begin{array}{l}\text { L1NI-B-1- } \\
\text { oviduct }\end{array}$ & $\begin{array}{l}\text { L2NI-B- } \\
\text { 2-ovary }\end{array}$ & $\begin{array}{l}\text { L2NI-B-2- } \\
\text { oviduct }\end{array}$ \\
\hline Ampicillin & 1 & 1 & $I$ & $\mathbf{R}$ & $R$ & $S$ & $\mathrm{~S}$ & $\mathrm{~S}$ & 1 & 1 & $S$ & $\mathrm{~S}$ & 1 & I & 1 & I \\
\hline Amoxycillin & । & $R$ & । & $R$ & $\mathrm{R}$ & । & $S$ & I & । & । & । & । & । & । & $\mathbf{R}$ & I \\
\hline Ceftiofur & S & S & $S$ & S & S & S & S & S & S & $S$ & $S$ & S & S & S & S & S \\
\hline Colistin & S & $S$ & $S$ & S & S & $S$ & $S$ & S & S & $S$ & $S$ & S & S & S & S & S \\
\hline Doxycycline & I & I & $S$ & $\mathbf{R}$ & $\mathbf{R}$ & $S$ & S & S & S & $S$ & $S$ & $\mathbf{R}$ & S & S & 1 & $S$ \\
\hline Enrofloxacin & S & $S$ & $S$ & S & S & S & S & S & S & $S$ & $S$ & I & S & S & S & S \\
\hline Gentamicin & S & S & $\mathrm{S}$ & S & S & S & S & S & S & S & S & S & S & S & S & S \\
\hline Neomycin & S & S & S & S & S & S & S & S & S & $S$ & $S$ & $\mathbf{R}$ & S & S & S & S \\
\hline Oxolinic acid & $S$ & $S$ & $S$ & $S$ & S & S & $S$ & S & $S$ & $\mathbf{R}$ & R & $\mathrm{R}$ & $\mathbf{R}$ & $S$ & S & S \\
\hline Tetracycline & S & S & $S$ & $\mathbf{R}$ & $\mathbf{R}$ & $S$ & S & S & S & $S$ & S & । & S & S & S & S \\
\hline Tylosin & $\mathrm{R}$ & $\mathrm{R}$ & $\mathrm{R}$ & $\mathrm{R}$ & $\mathrm{R}$ & $\mathrm{R}$ & $\mathrm{R}$ & $\mathrm{R}$ & $\mathrm{R}$ & $\mathrm{R}$ & $\mathrm{R}$ & $\mathrm{R}$ & $R$ & R & $\mathrm{R}$ & $\mathrm{R}$ \\
\hline Spectinomycin & S & S & $S$ & S & S & S & $S$ & S & S & $S$ & $S$ & S & S & S & S & S \\
\hline $\begin{array}{l}\text { Sulphamethoxazole + } \\
\text { trimethoprim }\end{array}$ & S & S & 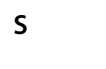 & $\mathbf{R}$ & $\mathbf{R}$ & $S$ & S & S & S & $S$ & $S$ & S & S & S & S & S \\
\hline
\end{tabular}

Each isolate ID is designated with letter " $\mathrm{L}$ " along with the number of layer flock and rearing farm - A (sampling at the peak of production) or B (sampling at the end of production) - number of sampled bird - organ of isolation - isolate number (in case when two isolates were collected from the same organ). Antibiotic resistance pattern of two isolates from the same bird are in bold letter and highlighted if they showed different of isolation - isolate number (in case when two isolates were collected from
sensitivity to antimicrobials used. S: sensitive, I intermediate, R: resistant 
not very high, it already provides an indication for the problem of antibiotic resistance in E. coli towards commonly used antimicrobials. In a recent report from China, $E$. coli isolates collected from chickens were sensitive to relatively newer antibiotics such as cephalosporin but MDR rate was as high as $80.25 \%$ [11]. The results from the present study further indicate that isolates collected from the same bird may not necessarily have identical antibiotic sensitivity profiles. Thus it can be suggested that testing of the antibiotic sensitivity profile from just one isolate per bird might not be enough to decide the most appropriate treatment.

\section{Conclusions}

Serotyping, antibiotic resistance test and genotypic fingerprinting of extraintestinal $E$. coli revealed that isolates exhibit high diversities within and between birds. As one bird can harbour different $E$. coli types an appropriate number of isolates should be considered for epidemiological studies and antibiotic sensitivity test.

\section{Abbreviations}

AMR: Antimicrobial resistance; APEC: Avian Pathogenic Escherichia coli; COS: Columbia agar supplemented with $5 \%$ sheep blood; MDR: Multidrug resistance; PCR: Polymerase chain reaction; PFGE: Pulsed-field gel electrophoresis

\section{Acknowledgements}

The support and collaboration from Austrian Poultry Veterinarians during the study are highly appreciated.

\section{Funding}

The authors do not have external funding for the study.

\section{Availability of data and materials}

The datasets supporting the conclusions of this article are included within the article and partial 16S rRNA gene sequence data are deposited in the European Nucleotide Archive.

\section{Authors' contributions}

$\mathrm{SP}, \mathrm{CH}, \mathrm{AZ}, \mathrm{MH}$ designed the study. $\mathrm{CH}$ and $\mathrm{AZ}$ were involved in necropsy and sampling. SP and BS performed PFGE. SP drafted the manuscript and BS, $\mathrm{CH}, \mathrm{AZ}, \mathrm{MH}$ contributed with their inputs. All authors have read and approved the final manuscript.

\section{Competing interests}

The authors declare that they have no competing interests.

\section{Consent for publication}

Not applicable.

\section{Ethics approval and consent to participate}

Sampling was performed during post mortem investigations and complies with national legislation (Tierversuchsgesetz - TVG 2012, §1). Furthermore, the study was performed in co-operation with veterinarians in charge of the respective farms who have agreements with farm owners for applying veterinary procedures.

\section{Author details}

'Department for Farm Animals and Veterinary Public Health, Clinic for Poultry and Fish Medicine, University of Veterinary Medicine, Veterinärplatz 1, 1210 Vienna, Austria. ${ }^{2}$ Department for Farm Animals and Veterinary Public Health, Institute of Milk Hygiene, Milk Technology and Food Science, University of Veterinary Medicine, Veterinärplatz 1, Vienna 1210, Austria.
Received: 8 March 2016 Accepted: 4 October 2016

Published online: 07 October 2016

\section{References}

1. Nolan LK, Barnes HJ, Vaillancourt JP, Abdul-Aziz T, Logue CM. Colibacillosis. In: Swayne DE, editor. Diseases of Poultry. 13th ed. Ames: John Wiley \& Sons, Inc; 2013. p. 751-805

2. Dhillon AS, Jack OK. Two outbreaks of colibacillosis in commercial caged layers. Avian Dis. 1996:40:742-6

3. Vandekerchove D, De HP, Laevens H, Pasmans F. Colibacillosis in caged layer hens: characteristics of the disease and the aetiological agent. Avian Pathol. 2004;33:117-25

4. Jordan FT, Williams NJ, Wattret A, Jones T. Observations on salpingitis, peritonitis and salpingoperitonitis in a layer breeder flock. Vet Rec. 2005;157:573-7.

5. Zanella A, Alborali GL, Bardotti M, Candotti P, Guadagnini PF, Martino PA, Stonfer M. Severe Escherichia coli 0111 septicaemia and polyserositis in hens at the start of lay. Avian Pathol. 2000;29:311-7.

6. Landman WJ, van Eck JH. The incidence and economic impact of the Escherichia coli peritonitis syndrome in Dutch poultry farming. Avian Pathol. 2015:44:370-8

7. Olsen RH, Bisgaard M, Christensen JP, Kabell S, Christensen H. Pathology and Molecular Characterization of Escherichia Coli associated With the Avian Salpingitis-Peritonitis Disease Syndrome. Avian Dis. 2016;60:1-7.

8. Srinivasan P. Balasubramaniam GA, Murthy TR, Balachandran P. Bacteriological and pathological studies of egg peritonitis in commercial layer chicken in Namakkal area. Asian Pac J Trop Biomed. 2013;3:988-94.

9. Dho-Moulin M, Fairbrother JM. Avian pathogenic Escherichia coli (APEC). Vet Res. 1999;30:299-316.

10. Ewers C, Jannsen T, Wieler LH. Avian pathogenic Escherichia coli (APEC). Berl Munch Tierarztl Wochenschr. 2003;116:381-95.

11. Dou X, Gong J, Han X, Xu M, Shen H, Zhang D, Huang L, Liu J, Zou J. Characterization of avian pathogenic Escherichia coli isolated in eastern China. Gene. 2016:576:244-8.

12. Paixao AC, Ferreira AC, Fontes $M$, Themudo $P$, Albuquerque $T$, Soares $M C$, Fevereiro M, Martins L, de Sa MI C. Detection of virulence-associated genes in pathogenic and commensal avian Escherichia coli isolates. Poult Sci. 2016. doi:10.3382/ps/pew087.

13. Kemmett K, Humphrey T, Rushton S, Close A, Wigley P, Williams NJ. A longitudinal study simultaneously exploring the carriage of APEC virulence associated genes and the molecular epidemiology of faecal and systemic E. coli in commercial broiler chickens. PLoS One. 2013;8:e67749.

14. Collingwood C, Kemmett K, Williams N, Wigley P. Is the concept of Avian Pathogenic Escherichia coli (APEC) as a single pathotype is fundamentally flawed? Frontiers in Veterinary Science. 2014;1:5. doi:10.3389/fvets.2014.00005.

15. Torpdahl M, Skov MN, Sandvang D, Baggesen DL. Genotypic characterization of Salmonella by multilocus sequence typing, pulsed-field gel electrophoresis and amplified fragment length polymorphism. J Microbiol Methods. 2005:63:173-84

16. Ewers C, Janssen T, Kiessling S, Philipp HC, Wieler LH. Molecular epidemiology of avian pathogenic Escherichia coli (APEC) isolated from colisepticemia in poultry. Vet Microbiol. 2004;104:91-101.

17. Landman WJ, Buter GJ, Dijkman R, van Eck JH. Molecular typing of avian pathogenic Escherichia coli colonies originating from outbreaks of E. coli peritonitis syndrome in chicken flocks. Avian Pathol. 2014;43:345-56.

18. Barbieri NL, de Oliveira AL, Tejkowski TM, Pavanelo DB, Matter LB, Pinheiro SR, Vaz TM, Nolan LK, Logue CM, de Brito BG, Horn F. Molecular characterization and clonal relationships among Escherichia coli strains isolated from broiler chickens with colisepticemia. Foodborne Pathog Dis. 2015;12:74-83.

19. Carroll NM, Jaeger EE, Choudhury S, Dunlop AA, Matheson MM, Adamson P, Okhravi N, Lightman S. Detection of and discrimination between grampositive and gram-negative bacteria in intraocular samples by using nested PCR. J Clin Microbiol. 2000;38:1753-7.

20. Bauer AW, Kirby WM, Sherris JC, Turck M. Antibiotic susceptibility testing by a standardized single disk method. Am J Clin Pathol. 1966:45:493-6.

21. Blanco JE, Blanco M, Mora A, Jansen WH, Garcia V, Vazquez ML, Blanco J. Serotypes of Escherichia coli isolated from septicaemic chickens in Galicia (northwest Spain). Vet Microbiol. 1998;61:229-35.

22. Zhao S, Maurer JJ, Hubert S, De Villena JF, McDermott PF, Meng J, Ayers S, English L, White DG. Antimicrobial susceptibility and molecular characterization of avian pathogenic Escherichia coli isolates. Vet Microbiol. 2005;107:215-24. 
23. Pasquali F, Lucchi A, Braggio S, Giovanardi D, Franchini A, Stonfer M, Manfreda G. Genetic diversity of Escherichia coli isolates of animal and environmental origins from an integrated poultry production chain. Vet Microbiol. 2015:178:230-7.

24. Trampel DW, Wannemuehler Y, Nolan LK. Characterization of Escherichia coli isolates from peritonitis lesions in commercial laying hens. Avian Dis. 2007; 51:840-4.

Submit your next manuscript to BioMed Central and we will help you at every step:

- We accept pre-submission inquiries

- Our selector tool helps you to find the most relevant journal

- We provide round the clock customer support

- Convenient online submission

- Thorough peer review

- Inclusion in PubMed and all major indexing services

- Maximum visibility for your research

Submit your manuscript at www.biomedcentral.com/submit 